\title{
Molecular-genetic analysis of genome incompatibility in wheat-rye hybrids
}

\author{
Tsvetkova N.V. ${ }^{1}$, Zykin P.A. ${ }^{1}$, Andreeva E.A. ${ }^{1,2}$, Hackauf B. ${ }^{3}$, Voylokov A.V. ${ }^{2}$ \\ ${ }^{1}$ St. Petersburg State University, St. Petersburg, Russia \\ ${ }^{2}$ Vavilov Institute of General Genetics RAS, St. Petersburg, Russia \\ ${ }^{3}$ Julius Kühn-Institut, Institute for Breeding Research on Agricultural Crops, Sanitz, Germany \\ *email: n.tswetkowa@spbu.ru
}

The post-zygotic reproductive isolation in plants is frequently based on the negative interaction of the parental genes involved in plant development. The interaction of genes is the basis of the canonical scheme (Bateson-Dobzhansky-Muller model), explaining the emergence and functioning of post-zygotic incompatibility. Linkage analysis of microsatellite loci, as well as genes of embryo lethality (Eml-R1) and hybrid dwarfness (Hdw-R1), was performed in hybrids of Chinese Spring wheat with recombinant inbred lines as well as interline rye hybrids (Tsvetkova et al., 2018). Eml-R1 and Hdw-R1 has been mapped proximally and distally of two closely linked EST-SSR markers, Xgrm0902 and Xgrm0959, on the rye chromosome 6R which contains a breakpoint of evolutionary translocation between the ancestral chromosomes of homeologous groups 6 and 3. We have performed bioinformatic search of candidate genes in this region using transcripts isolated from tis-sues of the inbred rye line L7 from Petergof genetic collection. The assembly of the transcripts was done with SpaDeS. Each of the ESTderived SSR markers Xgrm0959, Xgrm0902 and mRNA sequences were mapped on chromosome-scale genome assembly (Rabanus-Wallace et al., 2021). The area of chromosome 6 between the markers contains sequences for transcription factors of two types: bHLH and Myb. Sequence similarity search in wheat and barley genome databases revealed orthologs of rye genes SECCE6Rv1G0419580.1 (bHLH) and SECCE6Rv1G0427780.1 (Myb) annotated as genes participating in plant development. Future transcriptomic analysis of the mutant lines will allow to understand the role of supposed candidate genes in interspecies isolation. 